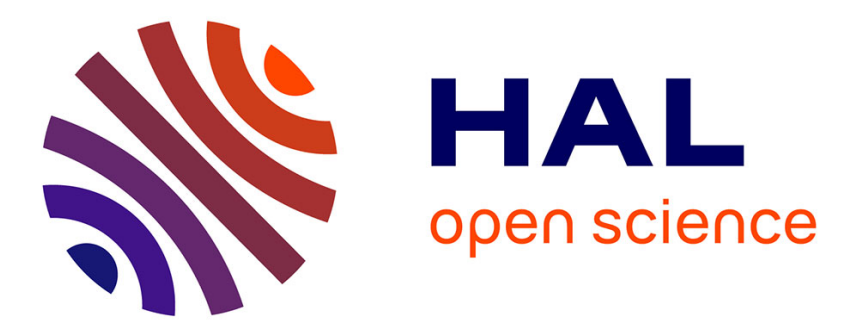

\title{
Dosage de traces d'éléments légers par activation par les photons $\gamma$ et les particules chargées
}

G. Cabane, Ch. Engelmann

\section{To cite this version:}

G. Cabane, Ch. Engelmann. Dosage de traces d'éléments légers par activation par les photons $\gamma$ et les particules chargées. Revue de Physique Appliquée, 1968, 3 (4), pp.365-372. 10.1051/rphysap:0196800304036500 . jpa-00242874

\section{HAL Id: jpa-00242874 https://hal.science/jpa-00242874}

Submitted on 1 Jan 1968

HAL is a multi-disciplinary open access archive for the deposit and dissemination of scientific research documents, whether they are published or not. The documents may come from teaching and research institutions in France or abroad, or from public or private research centers.
L'archive ouverte pluridisciplinaire HAL, est destinée au dépôt et à la diffusion de documents scientifiques de niveau recherche, publiés ou non, émanant des établissements d'enseignement et de recherche français ou étrangers, des laboratoires publics ou privés. 


\title{
DOSAGE DE TRAGES D’ÉLÉMENTS LÉGERS PAR AGTIVATION PAR LES PHOTONS $\gamma$ ET LES PARTIGULES GHARGÉES
}

\author{
Par G. CABANE et Ch. ENGELMANN, \\ Département de Métallurgie, G.E.N. de Saclay.
}

\begin{abstract}
Résumé. - Des radio-isotopes tels que ${ }^{11} \mathrm{C},{ }^{13} \mathrm{~N},{ }^{15} \mathrm{O},{ }^{18} \mathrm{~F}$, peuvent être formés par activation avec des photons ou des particules chargées et permettent ainsi les dosages des éléments légers à des teneurs variant de $10^{-6}$ à $10^{-9}$. Du fait de cette extrême sensibilité et de la possibilité de s'affranchir de toute contamination superficielle, ces méthodes d'analyse par activation sont irremplaçables pour les matériaux de très haute pureté, et elles ont déjà fourni des résultats ( $\mathrm{O}, \mathrm{C}$ et $\mathrm{N}$ dans $\mathrm{Si}, \mathrm{Fe}, \mathrm{Nb}$ par exemple) qui n'étaient accessibles par aucune autre méthode. Les applications, tant pour les semiconducteurs que pour les métaux, se développent grâce à la disponibilité croissante des cyclotrons et des accélérateurs linéaires. Si l'avance technique des équipes françaises dans ce domaine peut être conservée, ce sera un atout très important pour la mise au point de nouveaux matériaux semiconducteurs et pour le succès de nombreuses recherches de métallurgie physique.
\end{abstract}

Abstract. - Radio-isotopes such as ${ }^{11} \mathrm{C},{ }^{13} \mathrm{~N},{ }^{15} \mathrm{O},{ }^{18} \mathrm{~F}$ can be produced by photons or charged particle activation, and so allow the detection and analysis of light elements at levels of $10^{-6}$ to $10^{-9}$. Because of this extreme sensitivity and the possibility of getting rid of surface contamination, these types of activation analysis are unchallenged for very high purity materials, and indeed they already have given results (for $\mathrm{O}, \mathrm{C}, \mathrm{N}$ in $\mathrm{Si}, \mathrm{Fe}$ and $\mathrm{Nb}$ for example) that cannot be obtained by any other method. Applications for semiconductors as well as metals are mushrooming as cyclotrons and linear accelerators become progressively more accessible. If the technical advance of French teams can be maintained, this will be a very important factor for the development of new semiconductor materials and for the success of many studies in physical metallurgy.

Introduction. - Les réactions nucléaires ont trouvé un champ d'application particulièrement intéressant dans le domaine des analyses de traces. En effet, les noyaux des atomes des échantillons irradiés reçoivent des énergies considérables qu'ils réémettent sous forme de rayonnements divers lors du réarrangement nucléaire. Les caractéristiques de ces rayonnements dépendent de la structure physique du noyau bombardé, de la nature du projectile incident ainsi que de son énergie. Par conséquent, la mesure qualitative et quantitative du rayonnement émis permet de dénombrer la quantité de noyaux d'une espèce donnée contenue dans un milieu support donné.

L'analyse par activation a été développée en France par le travail de pionnier du Professeur Albert et de son équipe. Grâce à cette vigoureuse impulsion, les différentes techniques d'analyse utilisant l'activation par des neutrons, par des photons $\gamma$ ou par des particules chargées, ont été perfectionnées en France avec une nette avance sur les réalisations étrangères. Il faut s'en féliciter car les dosages de traces infimes, qui ont été ainsi rendus possibles, ont permis des progrès spectaculaires dans la préparation des métaux de haute pureté et dans le développement des recherches de métallurgie et de physique des solides, qui ont besoin de ces matériaux.

L'émission consécutive à l'irradiation peut se produire instantanément, c'est-à-dire dans un intervalle de temps équivalent à la durée de vie de l'état nucléaire créé, ou d'une façon retardée. Ce dernier type de radioactivité est alors caractérisé par la période de décroissance du noyau produit. C'est la base de la méthode qui sera exposée ci-dessous car les mesures $\mathrm{du}$ rayonnement émis au moment de la réaction nucléaire seront décrites dans un autre exposé par M. Amsel.

L'activation par les neutrons thermiques ou rapides permet le dosage de traces d'éléments de $Z$ supérieur à 10 ; ses nombreuses applications ont justifié en France comme à l'étranger son développement rapide, principalement à Grenoble. Avec la construction du laboratoire Pierre-Sue relié au réacteur « Osiris », on pourra disposer bientôt à Saclay de remarquables possibilités d'activation neutronique.

Nous décrirons seulement ici les méthodes d'activation par photons $\gamma$ ou particules chargées, qui sont particulièrement remarquables pour le dosage d'éléments légers qui, même à l'état de traces infimes, 
peuvent modifier profondément les propriétés des métaux et des semiconducteurs. On peut aussi activer ainsi des éléments à faible section de capture neutronique comme le zirconium et le plomb, et à l'inverse des éléments inclus dans un échantillon s'activant beaucoup par les neutrons : c'est le cas du dosage d'impuretés dans les terres rares, ou du dosage de ces terres rares elles-mêmes, c'est aussi le cas du dosage de l'oxygène dans l'uranium ou les actinides par activation avec les hélions 3 , seule particule utilisable pour activer les éléments légers à une énergie inférieure à celle qui provoque la fission.

Principes des analyses par activation. - Tous les métaux sont recouverts de couches d'oxyde plus ou moins nitruré; les contaminations superficielles par le carbone sont également très fréquentes et difficiles à supprimer totalement. C'est pourquoi, si on veut connaître la teneur de ces éléments dans la masse de l'échantillon, il est indispensable de se débarrasser des couches superficielles. L'activation avec comptage postérieur est la seule méthode qui permet cela, grâce à un décapage ou une abrasion suivant immédiatement l'irradiation (inversement, on peut naturellement doser la contamination superficielle et son extension en profondeur). Les impuretés des réactifs ou de toute autre origine apportées après l'irradiation n'ont plus aucune incidence perturbatrice puisqu'elles ne sont pas activées. Ainsi le dosage d'impureté à l'état de trace ultime, c'est-à-dire dans une gamme de teneurs de $10^{-9}$ à $10^{-6}$, est effectué avec le maximum de sécurité $\left.{ }^{1}\right)$.

Ces sensibilités ont été illustrées par la mise au point des méthodes de dosage des impuretés dans le béryllium [1].

L'AGtivation. - L'utilité pour l'analyse par activation des réactions nucléaires est fonction de leur section efficace et des grandeurs déterminant les possibilités de réaction : énergie ou $Q$ de la réaction, seuil et barrière coulombienne.

Si le bilan des masses est négatif, le seuil vaut :

$$
S=(Q) \frac{M+m}{M} \text {. }
$$

Si $Q$ est positif, le seuil est nul, mais il faut néanmoins franchir la barrière coulombienne, dont la hauteur croît avec le numéro atomique du matériau irradié. Ceci fait l'intérêt de l'activation par les hélions 3 pour lesquels les seuils sont toujours faibles.

Avec ces particules, on peut activer des éléments légers, l'oxygène par exemple, à des énergies inférieures aux barrières coulombiennes de la matrice, surtout si

(1) Pour ceux auxquels ces unités de concentration ne sont pas familières, rappelons qu'une limite de détection de l'oxygène de $10^{-9}$ correspond à $10^{13}$ atomes d'oxygène par $\mathrm{cm}^{3}$ de silicium. celle-ci est un élément lourd. Les hélions 3 présentent toutefois deux inconvénients :

- Comme les seuils sont très faibles, les réactions possibles avec les éléments légers sont nombreuses; il y a donc des interférences possibles lorsque l'impureté à doser n'est pas le seul élément léger dans le matériau à analyser.

- D'autre part, la pénétration des hélions 3 accélérés à 10-15 MeV est seulement de l'ordre de 70 à 140 microns pour l'aluminium, donc beaucoup moins pour un métal lourd ( $\mathrm{Nb}, \mathrm{Ta} . .$.$) . C'est excellent si on$ veut faire un dosage superficiel, mais si on désire connaître la teneur en impureté dans la masse, il faut décaper ou abraser après activation, ce qui rend aléatoire la profondeur activée restante, si elle n'est pas supérieure à quelques dizaines de microns.

Si l'énergie du rayonnement incident est assez élevée, la réaction nucléaire se produit et l'activité induite est :

$$
\left(\frac{\mathrm{d} N}{\mathrm{~d} t}\right)_{0}=N \int_{0}^{x} \varphi_{x} \sigma_{x} \mathrm{~d} x \cdot\left(1-\mathrm{e}^{-\lambda t}\right)
$$

$N \quad$ : nombre de noyaux activables dans l'échantillon : c'est la teneur recherchée, $\varphi_{x}$ et $\sigma_{x}$ : sont les flux et section efficace du rayonnement à la profondeur $x$ de l'échantillon (c'est-à-dire après un certain ralentissement),

$\left(1-\mathrm{e}^{-\lambda t}\right)$ : facteur représentant la décroissance d'activité au cours de l'irradiation.

Dans le cas des photons $\gamma$ qui sont peu absorbés, on peut, sans erreur importante, remplacer l'intégrale par $\varphi_{0} \sigma_{0}$, flux et section efficace $d u$ rayonnement incident.

Dans le cas des particules chargées qui se ralentissent rapidement dans le matériau à analyser, on ne peut plus faire la même approximation. Il est possible, par contre, de tracer expérimentalement la fonction d'activation en mesurant les activités de moniteurs placés devant et derrière des échantillons d'épaisseurs variées. Par cette méthode [2], on détermine 1'épaisseur équivalente, c'est-à-dire une épaisseur fictive dans laquelle l'activité spécifique caractéristique de l'impureté dosée serait constante dans l'échantillon, et égale à celle créée dans un échantillon infiniment mince, irradié dans le même faisceau :

$$
e=\frac{\int_{0}^{x} \varphi_{x} \sigma_{x} \mathrm{~d} x}{\varphi_{0} \sigma_{0}}
$$

Après cet étalonnage, on mesure facilement la teneur en impureté de tout échantillon :

$$
Q=\frac{N_{\mathrm{e}}}{N_{\mathrm{t}}} \frac{1}{e p S}
$$

$N_{\mathrm{e}}$ : activité déterminée dans l'échantillon, $N_{\mathrm{t}}$ : activité spécifique du témoin. 
A titre d'exemple, les épaisseurs équivalentes suivantes ont été déterminées pour le dosage de l'oxygène dans l'aluminium :

- Avec des protons, pour la réaction ${ }^{18} \mathrm{O}(\mathrm{p}, \mathrm{n})^{18} \mathrm{~F}$ : $e=640$ microns à $9 \mathrm{MeV}$ et 3400 microns à $19 \mathrm{MeV}$ ceci correspond à des produits $N_{\mathrm{t}} e \rho S$ que nous appelons activité par concentration massique unitaire, de $3500 \mathrm{cpm} / \mathrm{cm}^{2} \mathrm{ppm} \mathrm{O}_{2}$ à $9 \mathrm{MeV}$ et $7800 \mathrm{cpm} / \mathrm{cm}^{2}$ ppm $\mathrm{O}_{2}$ à $19 \mathrm{MeV}$ (pour une intensité de faisceau de $10 \mu \mathrm{A}$ et une durée d'irradiation d'environ deux périodes).

- Avec des particules $\alpha$ (de $44 \mathrm{MeV})$, pour la réaction ${ }^{16} \mathrm{O}(\alpha, \mathrm{pn})^{18} \mathrm{~F}$, on trouve :

$$
e=500 \text { microns. }
$$

Le comptage. - Les produits des réactions nucléaires avec les éléments légers sont presque toujours les noyaux ${ }^{18} \mathrm{~F}-{ }^{15} \mathrm{O}-{ }^{13} \mathrm{~N}-{ }^{11} \mathrm{C}$ qui sont des émetteurs de positrons, de périodes respectives $112 ; 2,1 ; 10$ et 20 minutes. Les échantillons étant enfermés dans de petites boîtes métalliques, les positrons s'y désintègrent en donnant deux photons émis dans des directions opposées. Deux compteurs situés de part et d'autre de l'échantillon et montés en coïncidence permettent d'enregistrer ce rayonnement avec un très faible bruit de fond.

Les périodes ci-dessus sont assez différentes pour que la décomposition des courbes de décroissance soit facile et précise.

Mais dans certains cas il apparaît d'autres activités de périodes voisines par suite de réactions nucléaires avec la matrice ou avec d'autres impuretés. Pour résoudre ces problèmes, on a mis au point plusieurs méthodes de séparation chimique rapides qui ont des rendements excellents et reproductibles.

Il subsiste un dernier type d'interférence : c'est celui qui est provoqué par des réactions nucléaires plus complexes produisant le même noyau à partir d'autres éléments; par exemple, on dose le carbone avec la réaction ${ }^{12} \mathrm{C}(\gamma, n){ }^{11} \mathrm{C}$, mais le même isotope ${ }^{11} \mathrm{C}$ peut être formé à partir de ${ }^{14} \mathrm{~N}$ ou ${ }^{16} \mathrm{O}$ si ces éléments sont abondants dans l'échantillon (dosage de carbone dans un oxyde par exemple) par :

$$
{ }^{14} \mathrm{~N}(\gamma, \mathrm{t}){ }^{11} \mathrm{C} \quad \text { et } \quad{ }^{16} \mathrm{O}(\gamma, \alpha \mathrm{n})^{11} \mathrm{C} \text {. }
$$

Toutefois, l'erreur introduite par ces réactions est de second ordre, car les seuils de ces réactions plus complexes sont plus élevés, et les sections efficaces jusqu'à l'énergie maximale utilisée sont beaucoup plus faibles.

MÉTHOdes UTILISANT L'AGTIVATION PAR LES PHOTONs $\gamma$. Activation aux photons $\gamma$. - Le faisceau d'électrons de l'accélérateur linéaire bombarde une cible de platine dans laquelle leur énergie cinétique est convertie en rayonnement $\gamma$ de freinage. Le spectre d'énergie des électrons est une raie, celui des photons produits est continu. Le choix de la cible de conversion semble très important. En ce qui concerne le rendement global de transformation, ce sont les éléments lourds qui donnent les meilleures valeurs. Une étude du problème de la cible s'impose néanmoins. En effet, la forme optimale du faisceau de rayons $\gamma$ dépend du type d'irradiation que l'on désire. Les divers matériaux ne donnent pas la même distribution spatiale de la densité volumique de rayonnement de freinage pour une zone d'énergie considérée. Une partie des expériences en cours a pour objet de définir respectivement la structure et la composition physique de la cible pour laquelle l'activation est maximale et homogène, compte tenu de l'impureté à doser et des dimensions d'échantillons.

Dosages de $\mathrm{C}, \mathrm{N}, \mathrm{O}$ et $\mathrm{F}$. - Ces quatre éléments donnent les isotopes radioactifs indiqués dans le tableau I, où l'on trouve également les réactions nu-

\section{TABLEAU I}

\begin{tabular}{|c|c|c|c|c|c|}
\hline $\begin{array}{l}\text { ÉLÉMENT } \\
\text { A }\end{array}$ & $\begin{array}{c}\text { RÉAGTION } \\
\text { NUGLÉAIRE } \\
\text { UTILISÉE }\end{array}$ & SeUIL & $\begin{array}{l}\text { Principales } \\
\text { RÉaGtions }\end{array}$ & SEUIL & PÉRIODES \\
\hline DOSER & POUR LE DOSAGE & $\mathrm{MeV}$ & D'INTERFÉRENGES & $\mathrm{MeV}$ & RADIOAGTIVES \\
\hline C & ${ }^{12} \mathrm{C}(\gamma, \mathrm{n}){ }^{11} \mathrm{C}$ & 18,7 & $\begin{array}{l}{ }^{14} \mathrm{~N}(\gamma, \mathrm{t})^{11} \mathrm{C} \\
{ }^{16} \mathrm{O}(\gamma, \alpha \mathrm{n})^{11} \mathrm{C}\end{array}$ & $\begin{array}{l}22,7 \\
26\end{array}$ & $\begin{array}{c}20,3 \mathrm{mn} \\
-\end{array}$ \\
\hline $\mathrm{N}$ & ${ }^{14} \mathrm{~N}(\gamma, n){ }^{13} \mathrm{~N}$ & 10,5 & ${ }^{16} \mathrm{O}(\gamma, \mathrm{t}){ }^{13} \mathrm{~N}$ & 25 & $10 \mathrm{mn}$ \\
\hline $\mathrm{O}$ & ${ }^{16} \mathrm{O}(\gamma, \mathrm{n}){ }^{15} \mathrm{O}$ & 15,7 & $\begin{array}{r}{ }^{19} \mathrm{~F}(\gamma, \text { tn }){ }^{15} \mathrm{O} \\
\left.{ }^{20} \mathrm{Ne}(\gamma, \propto n)\right)^{15} \mathrm{O}\end{array}$ & $\begin{array}{l}27,4 \\
20,4\end{array}$ & $\begin{array}{c}2,1 \mathrm{mn} \\
-\end{array}$ \\
\hline $\mathrm{F}$ & ${ }^{19} \mathrm{~F}(\gamma, \mathrm{n}){ }^{18} \mathrm{~F}$ & 10,5 & $\begin{array}{l}{ }^{20} \mathrm{Ne}(\gamma, \mathrm{d}){ }^{18} \mathrm{~F} \\
{ }^{23} \mathrm{Na}(\gamma, \alpha \mathrm{\alpha}){ }^{18} \mathrm{~F}\end{array}$ & $\begin{array}{l}21,1 \\
21\end{array}$ & $1,7 \mathrm{~h}$ \\
\hline
\end{tabular}

Pringipales réagtions photonugléaires intervenant dans les dosages de $\mathrm{G}, \mathrm{N}, \mathrm{O}$ et $\mathrm{F}$ 
cléaires d'interférences et les divers seuils, c'est-à-dire les énergies minimales à partir desquelles les réactions sont possibles.

L'énergie d'irradiation ne peut pas être quelconque au-dessus du seuil de la réaction utilisée pour les analyses. Il existe en effet toujours des réactions sur d'autres éléments dont le résultat est de créer le même isotope radioactif que celui servant pour les dosages.

Par exemple, l'oxygène peut également donner de l'azote 13 et du carbone 11 au moyen des réactions :

$$
{ }^{16} \mathrm{O}(\gamma, \mathrm{t})^{13} \mathrm{~N} \text { et }{ }^{16} \mathrm{O}(\gamma, \alpha \mathrm{n})^{11} \mathrm{G}
$$

interférant par conséquent avec les analyses d'azote et de carbone si l'énergie d'irradiation est très supérieure à leurs seuils.

Ces réactions photonucléaires d'interférences sont actuellement étudiées. Leurs seuils, pour les éléments légers du moins, sont dans la plupart des cas supérieurs à ceux des réactions $(\gamma, n)$.

Les dosages non destructifs des quatre impuretés ci-dessus sont possibles dans certains matériaux [3]. On peut trouver quelques exemples concrets dans le tableau II.

Dans le cas général, on peut toujours avoir recours aux procédés de séparations chimiques après irradiation et avant comptage. Pour le fluor, il ne semble pas y avoir de grandes difficultés. Par contre, la séparation des trois autres impuretés pose de nombreux problèmes.

Afin de mettre au point une méthode d'application très générale de dosage de $\mathrm{C}, \mathrm{N}$ et $\mathrm{O}$ à l'état de traces dans les matériaux de très haute pureté, on étudie des méthodes de séparations rapides de $\mathrm{N}$ et $\mathrm{O}$ d'une part, ou de $\mathrm{G}$ et $\mathrm{N}$ : les durées totales de séparation sont de l'ordre de $5 \mathrm{mn}$ pour la première méthode et $20 \mathrm{mn}$ pour la seconde.

Dans ces conditions, en irradiant les échantillons pendant des durées de l'ordre de deux périodes dans un faisceau d'intensité moyenne $100 \mu \mathrm{A}$, on peut doser les quantités minimales portées dans le tableau III; c'est-à-dire en activant des échantillons de plusieurs grammes, ce qui est parfaitement possible, on peut doser ces impuretés à des teneurs de quelque $10^{-3} \mathrm{ppm}$.

Quand on pense qu'une analyse simultanée d'oxygène et d'azote à de telles concentrations dure à peine $10 \mathrm{mn}$, on entrevoit toutes les possibilités de ces méthodes dans le domaine des analyses de traces.

Autres impuretés dosables. - D'autres impuretés sont également dosables. On peut citer par exemple le plomb dans les poussières et les milieux biologiques à des teneurs relatives de $10^{-7}$.

\section{TABLEAU II}

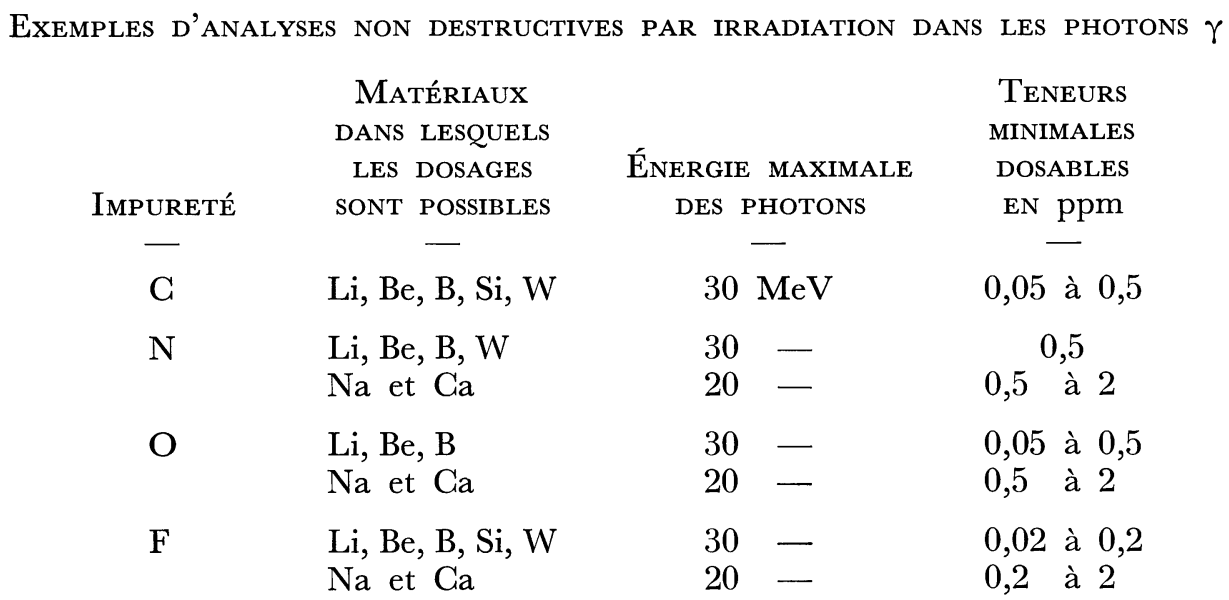

On suppose que la durée d'irradiation est d'une période et que le poids de l'échantillon est de $1 \mathrm{~g}$, le courant moyen du faisceau d'électrons étant de $100 \mu \mathrm{A}$.

\section{TABLEAU III}

Quantités minimales dosables de $\mathrm{C}, \mathrm{N}$, O et F par irRadiation dans les photons $\gamma$

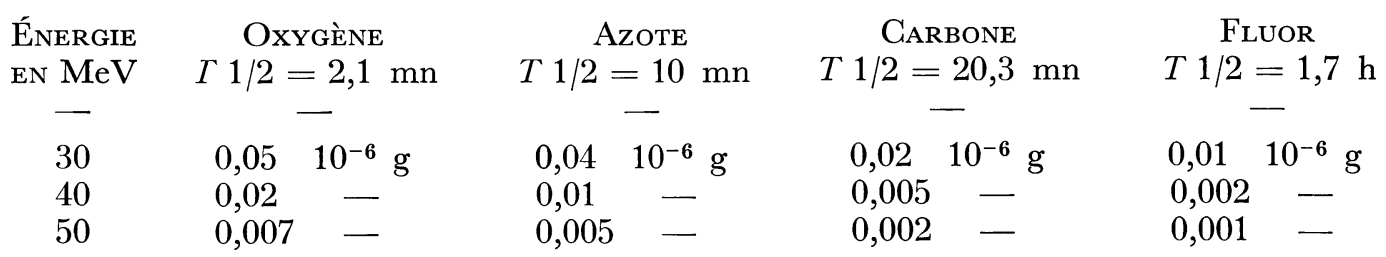


MÉTHODES UTILISANT L'AGTIVATION PAR DES PARTIGULES GHARGÉES. - Les particules utilisées pour ces expériences sont $\mathrm{d}, \mathrm{p},{ }^{3} \mathrm{He}$ et ${ }^{4} \mathrm{He}$. On trouvera dans le tableau IV les principales réactions nucléaires exploitables à des fins analytiques pour les impure- tés $\mathrm{B}, \mathrm{G}, \mathrm{N}$ et $\mathrm{O}$, leurs seuils et les réactions d'interférences les plus importantes.

Des dosages non destructifs sont ici également possibles [4]. Quelques exemples ayant fait l'objet de nos propres expériences sont donnés dans le tableau $\mathrm{V}$.

TABLEAU IV

Principales réagtions nugléaires aux partigules ghargées $\mathrm{p}, \mathrm{d},{ }^{3} \mathrm{He}$ et ${ }^{4} \mathrm{He}$ Utilisées pour les dosages de $\mathrm{B}, \mathrm{C}, \mathrm{N}$ et $\mathrm{O}$

\begin{tabular}{|c|c|c|c|c|c|}
\hline $\begin{array}{c}\text { ÉLÉMENT } \\
\text { A } \\
\text { DOSER } \\
\end{array}$ & $\begin{array}{c}\text { RÉAGTION NUGLÉAIRE } \\
\text { UTILISÉE } \\
\text { POUR LE DOSAGE } \\
\text { - }\end{array}$ & $\begin{array}{l}\text { SEUIL } \\
\text { EN } \\
\text { MeV } \\
-\end{array}$ & $\begin{array}{c}\text { Principales } \\
\text { RÉACTIONS } \\
\text { D'INTERFÉRENCES } \\
-\end{array}$ & $\begin{array}{l}\text { SEUIL } \\
\text { EN } \\
\mathrm{MeV} \\
-\end{array}$ & $\begin{array}{c}\text { PÉRIODES } \\
\text { RADIOAGTIVES } \\
-\end{array}$ \\
\hline B & $\begin{array}{l}{ }^{11} \mathrm{~B}(\mathrm{p}, \mathrm{n})^{11} \mathrm{C} \\
{ }^{10} \mathrm{~B}(\mathrm{~d}, \mathrm{n})^{11} \mathrm{C} \\
{ }^{11} \mathrm{~B}(\mathrm{~d}, 2 \mathrm{n})^{11} \mathrm{C}\end{array}$ & $\begin{array}{l}0 \\
5,90\end{array}$ & $\begin{array}{l}{ }^{12} \mathrm{C}(\mathrm{p}, \mathrm{pn}){ }^{11} \mathrm{C} \\
{ }^{14} \mathrm{~N}(\mathrm{p}, \alpha)^{11} \mathrm{C} \\
{ }^{14} \mathrm{~N}(\mathrm{~d}, \alpha \mathrm{n})^{11} \mathrm{C} \\
{ }^{12} \mathrm{C}(\mathrm{d}, \mathrm{t})^{11} \mathrm{C}\end{array}$ & $\begin{array}{r}20,3 \\
3,12 \\
5,88 \\
14,54\end{array}$ & $\begin{array}{c}20,3 \mathrm{mn} \\
- \\
-\end{array}$ \\
\hline G & $\begin{array}{l}{ }^{13} \mathrm{C}(\mathrm{p}, \mathrm{n}){ }^{13} \mathrm{~N} \\
{ }^{12} \mathrm{C}\left({ }^{3} \mathrm{He}, \alpha\right){ }^{11} \mathrm{C} \\
{ }^{12} \mathrm{C}(\alpha, \alpha \mathrm{n}){ }^{11} \mathrm{C}\end{array}$ & $\begin{array}{r}0 \\
25\end{array}$ & $\begin{array}{l}{ }^{14} \mathrm{~N}(\mathrm{p}, \mathrm{pn})^{13} \mathrm{~N} \\
{ }^{16} \mathrm{O}(\mathrm{p}, \alpha)^{13} \mathrm{~N} \\
{ }^{10} \mathrm{~B}\left({ }^{3} \mathrm{He}, \mathrm{pn}\right)^{11} \mathrm{C} \\
{ }^{9} \mathrm{Be}\left({ }^{3} \mathrm{He}, \mathrm{n}\right)^{11} \mathrm{C} \\
{ }^{9} \mathrm{Be}(\alpha, 2 n){ }^{11} \mathrm{C} \\
{ }^{10} \mathrm{~B}(\alpha, t)^{11} \mathrm{C} \\
{ }^{11} \mathrm{~B}(\alpha, \mathrm{tt})^{11} \mathrm{C}\end{array}$ & $\begin{array}{c}11,3 \\
5,5 \\
0 \\
0 \\
18,8 \\
15,6 \\
30,8\end{array}$ & $\begin{array}{c}10,0 \mathrm{mn} \\
- \\
20,3 \mathrm{mn} \\
= \\
= \\
-\end{array}$ \\
\hline $\mathrm{N}$ & $\begin{array}{l}{ }^{14} \mathrm{~N}(\mathrm{p}, \alpha){ }^{11} \mathrm{C} \\
{ }^{14} \mathrm{~N}(\mathrm{~d}, \mathrm{n}){ }^{15} \mathrm{O}\end{array}$ & 3,12 & $\begin{array}{l}{ }^{11} \mathrm{~B}(\mathrm{p}, \mathrm{n}){ }^{11} \mathrm{C} \\
\left.{ }^{12} \mathrm{G}(\mathrm{p}, \mathrm{pn})\right)^{11} \mathrm{C} \\
{ }^{16} \mathrm{O}(\mathrm{d}, \mathrm{t})^{15} \mathrm{O}\end{array}$ & $\begin{array}{l}3,02 \\
20,3 \\
10,6\end{array}$ & $\begin{array}{r}20,3 \mathrm{mn} \\
20,3 \mathrm{mn} \\
2,1 \mathrm{mn}\end{array}$ \\
\hline $\mathrm{O}$ & $\begin{array}{l}{ }^{18} \mathrm{O}(\mathrm{p}, \mathrm{n}){ }^{18} \mathrm{~F} \\
{ }^{16} \mathrm{O}\left({ }^{3} \mathrm{He}, \mathrm{p}\right)^{18} \mathrm{~F} \\
{ }^{16} \mathrm{O}(\alpha, \mathrm{d})^{18} \mathrm{~F}\end{array}$ & $\begin{array}{l}2,6 \\
0 \\
20,4\end{array}$ & $\begin{array}{l}{ }^{19} \mathrm{~F}(\mathrm{p}, \mathrm{pn}){ }^{18} \mathrm{~F} \\
{ }^{19} \mathrm{~F}\left({ }^{3} \mathrm{He}, \alpha\right)^{18} \mathrm{~F} \\
{ }^{19} \mathrm{~F}(\alpha, \alpha \mathrm{n})^{18} \mathrm{~F}\end{array}$ & $\begin{array}{c}11 \\
0 \\
12,6\end{array}$ & $\begin{array}{c}1,7 \mathrm{~h} \\
-\end{array}$ \\
\hline
\end{tabular}

TABLEAU $\mathrm{V}$

EXEMPLES D'ANALYSES NON DESTRUGTIVES PAR IRRADIATION DANS LES PARTIGULES GHARGÉES

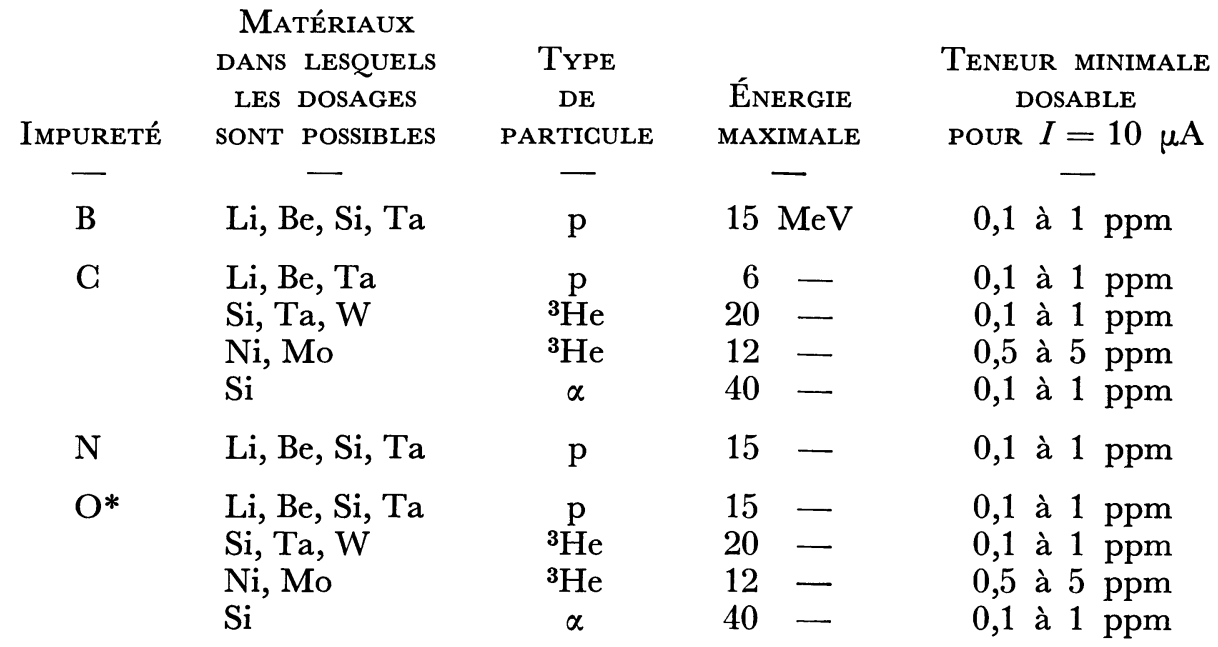

*Il s'agit de l'oxygène total. Pour l'isotope 18, la sensibilité est 500 fois meilleure aux protons. 
TABLEAU VI

Quantités minimales dosables de $\mathrm{B}, \mathrm{C}, \mathrm{N}$ et $\mathrm{O}$ Par irRadiation dans les partigules Ghargées

\begin{tabular}{ccccc} 
ÉLÉment & $\mathrm{p}$ & $\mathrm{d}$ & ${ }^{3} \mathrm{He}$ & $\alpha$ \\
A & $E=20 \mathrm{MeV}$ & $E=20 \mathrm{MeV}$ & $E=20 \mathrm{MeV}$ & $E=44 \mathrm{MeV}$ \\
DOSER & $I=10 \mu \mathrm{A}$ & $I=10 \mu \mathrm{A}$ & $I=10 \mu \mathrm{A}$ & $I=10 \mu \mathrm{A}$ \\
\hline- & - & - & - & - \\
$\mathrm{B}$ & $0,001 \mathrm{ppm}$ & $0,0001 \mathrm{ppm}$ & - & - \\
$\mathrm{G}$ & - & - & $0,001 \mathrm{ppm}$ & $0,01 \mathrm{ppm}$ \\
$\mathrm{N}$ & $0,0005 \mathrm{ppm}$ & $0,0002 \mathrm{ppm}$ & - & - \\
$\mathrm{O}$ & $0,01 \mathrm{ppm}$ & - & $0,001 \mathrm{ppm}$ & $0,001 \mathrm{ppm}$
\end{tabular}

Le champ d'application est très vaste, particulièrement pour les analyses des couches minces, puisque tous les dosages sont non destructifs avec des sensibilités qui peuvent atteindre $10^{-9} \mathrm{~g} . \mathrm{cm}^{2}$.

$\mathrm{Si}$ les isotopes radioactifs sont séparés par des procédés chimiques après irradiation du type mentionné ci-dessus, on peut doser des teneurs très faibles. Le tableau VI contient quelques valeurs de quantités minimales dosables par irradiation dans les diverses particules.

Il faut enfin insister sur la spécificité isotopique de certaines réactions. Par exemple, avec des protons, on peut doser l'oxygène 18 à des teneurs de quelque $10^{-5} \mathrm{ppm}$, avec les deutons le bore 10 à des niveaux de $10^{-4} \mathrm{ppm}[5]$.

Equipements disponibles en France. - L'analyse par activation par les photons et les particules chargées nécessite des accélérateurs puissants, équipements lourds qui ne sont pas à la portée de la plupart des laboratoires métallurgiques. C'est pourquoi ce type d'analyse doit être réservé pour quelques années encore à des étalonnages d'autres méthodes relatives plus classiques, ou à la solution de quelques problèmes particuliers.

Mais les physiciens de tous les pays reportent progressivement leur intérêt de l'étude des noyaux à celle des nucléons. Par suite, les accélérateurs de petite ou moyenne énergie deviennent de plus en plus disponibles pour d'autres usages.

Pour utiliser ces possibilités, le laboratoire d'analyse par activation de Saclay a été relié par pneumatiques aux dispositifs d'irradiation installés sur trois accélérateurs. Ce sont :

- L'accélérateur linéaire qui délivre un courant d'électrons de $50 \mu \mathrm{A}$ moyen, et d'énergie réglable entre 30 et $45 \mathrm{MeV}$;

- Le cyclotron à énergie fixe qui est utilisé pour l'activation avec les $\alpha(44 \mathrm{MeV}, 5 \mu \mathrm{A})$ et pourrait aussi fonctionner avec des deutons;
- Le cyclotron à énergie variable que nous commençons à utiliser soit avec un faisceau de protons d'énergie réglable jusqu'à $24 \mathrm{MeV}$, soit avec un faisceau d'hélions 3 jusqu'à $35 \mathrm{MeV}$.

Les temps de disponibilité des faisceaux sont très variables, et souvent imprévisibles.

Grâce à l'amabilité de M. le Professeur Sarazin et de $\mathbf{M}$. Tousset, nous avons pu également faire quelques activations par des deutons avec le cyclotron de l'Institut de Physique Nucléaire de Lyon.

En regard de ces possibilités, il est intéressant de rappeler qu'aux États-Unis de nombreux laboratoires se sont équipés d'accélérateurs pour développer ces techniques d'analyse par activation mises au point en France. On peut compter maintenant 5 accélérateurs linéaires en fonctionnement, 1 commandé et 1 en construction, 3 cyclotrons de grande taille et sans doute plusieurs cyclotrons « de table », et enfin 3 Van de Graaff de 2 à $5 \mathrm{MeV}$.

Applications actuelles. - 1 . Nous dosons $\mathrm{O}, \mathrm{C}, \mathrm{N}$ dans des métaux nucléaires, ainsi que dans du fer et du niobium. Ces dernières analyses rentrent dans le cadre du contrat D.G.R.S.T. de M. Dautreppe. Il est bon de noter que l'analyse par activation permet pour la première fois d'étalonner les pics de frottement interne aux teneurs en impureté interstitielle inférieures à $10 \mathrm{ppm}$; on peut ainsi beaucoup mieux identifier les interactions entre impuretés différentes ou entre impuretés et défauts d'irradiation.

2. Le dosage de traces $(0,1$ à $10 \mathrm{ppm})$ d'oxygène et de carbone dans le sodium présente un grand intérêt pour le développement des réacteurs à neutrons rapides; les mêmes techniques d'activation par photons et de séparation chimique peuvent être utilisées pour les dosages de $\mathrm{O}, \mathrm{C}, \mathrm{N}$ et $\mathrm{F}$ dans les autres métaux alcalins et alcalino-terreux et certains de leurs composés.

3. Le troisième problème analytique important pour la métallurgie nucléaire est le dosage de traces de bore $(0,1$ à $10 \mathrm{ppm})$ dans les aciers inoxydables et les 
alliages de nickel étudiés pour le gainage des éléments combustibles. L'analyse par activation offre la possibilité de doser séparément ${ }^{10} \mathrm{~B}$ et ${ }^{11} \mathrm{~B}$ et, par suite, d'apprécier la proportion de ${ }^{10} \mathrm{~B}$ qui a été transformée en hélium par irradiation neutronique.

4. L'industrie des semiconducteurs est actuellement le premier client des analyses par activation, pour les dosages de traces de $\mathrm{O}, \mathrm{C}$ et $\mathrm{N}$ dans $\mathrm{Si}, \mathrm{Ge}$ et AsGa. Il faut souligner que dans tous les pays où l'on se préoccupe d'améliorer la productivité dans la fabrication des cristaux semiconducteurs et de réaliser de meilleurs détecteurs de rayonnement, le problème de dosage d'impuretés se pose à des niveaux de teneurs inférieures aux limites de sensibilité des méthodes classiques, de la spectrographie dans l'infrarouge et de la spectroscopie de masse.

Applications envisagées. - 1. Pour des études fines de diffusion, nous avons construit une machine d'abrasion qui permet d'enlever des couches de très faible épaisseur (jusqu'à 1 micron) de façon reproductible. Ceci permet d'envisager la détermination des gradients de concentration en impureté ou dopant sur des profondeurs de 10 à 1000 microns. Quelques essais ont été faits dans ce sens pour l'oxygène dans le silicium.

2. Avec le rayonnement $\beta^{+}$de ${ }^{18} \mathrm{~F}$ et peut-être de ${ }^{11} \mathrm{C}$, on peut envisager une autoradiographie. Cette technique a permis à Condit à Berkeley [6] de mettre en évidence la diffusion intergranulaire de l'oxygène daris la magnésie. Le premier problème à attaquer serait certainement l'étude de la pollution des joints de grains par l'oxygène.

3. L'élément léger le plus important en métallurgie, même aux très faibles concentrations, c'est assurément l'hydrogène. La seule méthode possible pour le doser par activation utilise la réaction ${ }^{1} \mathrm{H}(\mathrm{t}, \gamma)^{4} \mathrm{He}$ qui provoque une émission de $\gamma$ très énergique de $19 \mathrm{MeV}$ facilement identifiable. Mais ceci rentre dans le cadre des techniques qui sont décrites ci-après par M. Amsel.

4. A des énergies inférieures à $10 \mathrm{MeV}$, les photons $\gamma$ ne peuvent plus arracher de neutrons, mais ils peuvent encore produire des transitions d'isomérie nucléaire, notées $\left(\gamma, \gamma^{\prime}\right)[7]$.

Ces réactions permettent d'effectuer des dosages sans interférences dans de nombreux cas :

- Cd dans Zn, Hf dans Zr,

- Au, Pt, Ir, Ag dans les minerais de métaux rares,

- impuretés dans les corps organiques (hydrocarbures),

- éléments lourds dans les milieux biologiques.

5. Certaines impuretés dans les cristaux semiconducteurs peuvent se trouver en position de substitution ou d'insertion. Pour distinguer ces deux positions, on peut penser à utiliser les phénomènes de canalisation grâce auxquels la pénétration du rayonnement $(\mathrm{p}, \mathrm{d}$ ou $\alpha$ ), et par suite l'activation, est beaucoup plus importante pour certaines orientations du faisceau par rapport au monocristal irradié. Ce phénomène doit être beaucoup plus net pour une impureté en insertion (qui bouche un canal) que pour une impureté en substitution.

6. Les interférences des réactions nucléaires obligent souvent à recourir à des séparations chimiques. Malgré les succès obtenus dans de nombreux cas importants, il est souhaitable d'éviter cette étape à cause de la perte de sensibilité qu'entraîne le délai de la séparation chimique, et plus encore de l'incertitude d'un rendement de réaction qui n'est pas toujours strictement reproductible.

La perspective la plus intéressante dans ce sens nous paraît être de séparer les produits des réactions nucléaires par un champ électromagnétique. Gela revient à associer à l'accélérateur un séparateur d'isotopes; on bénéficierait ainsi en même temps de la grande sensibilité de l'analyse par activation et du coefficient multiplicateur considérable de l'émulsion photographique ou des photomultiplicateurs d'électrons.

D'autres usages importants attendent la libération des cyclotrons : chargements en hélium ou hydrogène, fabrication d'isotopes qu'on ne peut pas obtenir avec les neutrons, etc.

Problèmes de développement. - Comme par le passé, nous espérons que le C.E.A. continuera à financer les investissements importants que représentent les dispositifs d'irradiation, la remise en état du cyclotron à énergie fixe et l'entretien en général de ces accélérateurs. Mais le développement de l'analyse par activation avec les accélérateurs se heurte à deux obstacles:

Tout d'abord le nombre d'heures de disponibilité des faisceaux de particules est très insuffisant. Les programmes d'études de physique nucléaire laissent très peu de temps libre pour d'autres expérimentations, et les arrêts imprévus dus à des pannes du matériel sont d'autant plus graves que les périodes allouées d'utilisation sont plus courtes et plus espacées.

Par ailleurs, le nombre d'utilisateurs soit pour la mise au point des techniques analytiques, soit pour l'exploitation, augmente régulièrement, ce qui rend de plus en plus sensible le manque de temps d'utilisation des faisceaux de particules.

Ce problème recevra un début de solution lorsque le cyclotron à énergie fixe sera utilisé à plein temps pour l'analyse par activation (fin 1969) et lorsque la majeure partie des études de physique seront transférées de l'accélérateur de $45 \mathrm{MeV}$ à celui de $300 \mathrm{MeV}$. Mais l'analyse par activation avec les accélérateurs ne pourra trouver un développement normal qu'à la création du Centre d'Analyse par activation du C.N.R.S. à Orléans, où M. le Professeur Albert compte avoir un cyclotron à énergie variable et peutêtre un accélérateur linéaire.

D'autre part, le développement de ces techniques pose un problème de main-d'œuvre, non pas tant 
pour les analyses proprement dites que pour l'entretien et le fonctionnement des accélérateurs ainsi que pour l'étude et la réalisation des dispositifs d'irradiation.

Il est important et urgent de trouver des solutions à ces problèmes. La qualité d'une fabrication est toujours liée à la sensibilité et à la rigueur des contrôles. De même le développement des analyses par activation des traces d'éléments légers dans les métaux et les semiconducteurs est la condition impérative de l'amélioration des produits et de la mise au point de matériaux nouveaux.

\section{BIBLIOGRAPHIE}

[1] Petit (J. F.), Schaub (B.) et Engelmann (Ch.), Fusion de zone et analyse par activation, B.I.S.T., $\mathrm{n}^{\circ} 62$ (juin 1962).

Petit (J. F.) et Engelmann (Ch.), Dosage de traces d'impuretés dans le béryllium par des méthodes non destructives. Production and use of short lived radio-isotopes from reactors, IAEA, Vienne (1963).

ENGELMANN (Ch.), Analyse par activation du béryllium de très haute pureté obtenu par fusion de zone. Colloque sur la fusion de zone et de cristallisation basaltique, Errlangen (1963).

ENGELmanN (Ch.), Gossetr (J.) et LoEUILLET (Mlle M.), Analyse par activation de routine de quelques impuretés courantes du béryllium, Bull. Soc. Chim., France, 1965, 544.

[2] Engelmann (Ch.), Sur l'utilisation des particules $\alpha$ pour le dosage de l'oxygène et du carbone, $C . R$. Acad. Sci., 1964, 258, 4279.

ENGELMANN (Ch.), Analyse par activation aux particules chargées $\alpha$ et $\mathrm{p}$ de quelques éléments légers dans divers métaux. Congrès sur les méthodes radiochimiques d'analyse, AIEA-I-405, Salzbourg (1964).

[3] Albert (Ph.), Engelmann (Ch.), May (S.) et PETIT (J. F.), Analyse par activation d'oxygène, de carbone et d'azote au moyen de la réaction $(\gamma, \mathrm{n})$, C. R. Acad. Sci., 1962, 254, 119.

Engeimann (Ch.), Dosage de l'oxygène, du carbone, de l'azote et de quelques autres impuretés dans le béryllium, le calcium, le sodium et le bore par activation aux rayons $\gamma$. Congrès sur les méthodes radiochimiques d'analyse, AIEA-I-341, Salzbourg (1964).

ENGELMANN (Ch.), Emploi de particules autres que les neutrons en analyse par activation, Rapport C.E.A. 2559 (1964).
[4] Engelmann (Ch.), Gosset (J.) et Lafuilitet (Mlle M.), Analyse par activation de quelques métalloïdes à l'état de traces dans le béryllium ultra-pur. Conférence internationale sur la métallurgie du béryllium, Grenoble (mai 1965).

ENGELMANN (Ch.), Gosseit (J.) et LGUILIET (Mlle M.), Possibilités actuelles de dosages par activation de traces de métalloïdes dans les métaux réfractaires. Communication aux Journées métallurgiques d'automne, 11-15 octobre 1965, Paris, Mém. Sci. de Rev. Métal., 1966, 12, LXIII.

ENGELMANN (Ch.), Utilisation des photons $\gamma$ et des particules chargées pour les dosages de très faibles traces de $\mathrm{B}, \mathrm{C}, \mathrm{N}$ et $\mathrm{O}$. Communication à la Journée de Chimie Analytique, Paris, 1965, Bull. Soc. Chim. France, 1967, 7, 2316.

[5] Engelmann (Ch.) et Cabane (G.), Analyse des métallö̈des par activation avec des photons et des particules chargées, Proceedings international conference "Modern Trends in Activation Analysis", College Station, Texas (1965).

ENGELMANN (Ch.), Analyses de très faibles traces d'éléments légers dans les matériaux de très haute pureté par activation dans les photons $\gamma$ et les particules chargées. Communication au First IMR International Symposium "Trace Characterization Chemical and Physical", Gaithersburgh (1966), Int. Journ. Appl. Rad. and Isot., 1967, 18, 569-578.

[6] Hoct (J. B.) et CondiT (Rh.), Oxygen 18 diffusion in surface defects on $\mathrm{MgO}$ as revealed by proton activation, Materials Science Research, vol. 3, Plenum Press (1966).

[7] Engelmann (Ch.) et JÉROME (D. Y.), Application de l'isomérie nucléaire au dosage non destructif de certains éléments par activation dans les photons $\gamma$ de 4 à $8 \mathrm{MeV}$. Communication au Second International Meeting on "Practical Aspects of Activation Analysis with Charged Particles", Liège, 21-22 septembre 1967. 